\title{
Stability of Finite-Difference Models Containing Two Boundaries or Interfaces
}

\author{
By Lloyd N. Trefethen*
}

\begin{abstract}
It is known that the stability of finite-difference models of hyperbolic initial-boundary value problems is connected with the propagation and reflection of parasitic waves. Here the waves point of view is applied to models containing two boundaries or interfaces, where repeated reflection of trapped wave packets is a potential new source of instability. Our analysis accounts for various known instability phenomena in a unified way and leads to several new results, three of which are as follows. (1) Dissipativity does not ensure stability when three or more formulas are concatenated at a boundary or internal interface. (2) Algebraic "GKS instabilities" can be converted by a second boundary to exponential instabilities only when an infinite numerical reflection coefficient is present. (3) "GKS-stability" and " $P$-stability" can be established in certain problems by showing that the numerical reflection coefficient matrices have norm less than one.
\end{abstract}

0. Introduction. Hyperbolic systems of partial differential equations admit solutions which behave locally like waves moving along characteristics. When such a system is modeled numerically by finite differences or finite elements, the result is a dispersive medium that may admit additional parasitic wave modes with wave-lengths on the scale of the discretization. Energy associated with these parasitic waves travels at a group velocity that is unrelated to the characteristics of the original system [25], [30]. However, the behavior of such waves has a decisive effect on stability.

For finite-difference models of linear hyperbolic problems with a single spatial boundary, a stability theory was developed around 1970 by Kreiss, Osher, and others [10], [18]. In earlier papers we have shown that this theory can be naturally stated in terms of dispersive wave propagation [26], [27]. To summarize: if a boundary with homogeneous boundary conditions can emit a radiated wave in the absence of any incident waves, i.e., a wave with group velocity pointing into the interior of the domain, then it is unstable. If it has an infinite reflection coefficient for waves at some frequency, a stronger condition, then it is more severely unstable.

This paper applies wave propagation ideas to investigate stability for one-dimensional linear finite-difference models with two or more boundaries or internal interfaces. The most basic example of such a model is a discrete approximation to an equation whose spatial domain is an interval such as $[0,1]$. Another example is a model of a problem featuring discontinuous coefficients, e.g., wave propagation in a discontinuously stratified medium [4]. A third is a numerical scheme employing local

Received October 26, 1984.

1980 Mathematics Subject Classification. Primary 65M10.

* Research was supported in part by the U. S. Department of Energy under contract DE-AC02-76ER03077-V and in part by the National Aeronautics and Space Administration under NASA Contract No. NAS1-17070 while the author was in residence at the Institute for Computer Applications in Science and Engineering, NASA Langley Research Center, Hampton, VA 23665. 
mesh refinement to improve accuracy, in which various interfaces between fine and coarse grids will be present [2], [5]. A fourth is any model with a composite numerical boundary or interface, such as a fourth-order difference model on $[0, \infty)$ that has a five-point stencil, and which therefore requires one numerical boundary condition at $j=0$ and another at $j=1$ [15]. Such a model can be viewed as containing two interfaces separated by a single grid point, and we will show that this view may be useful for stability analysis.

Any multi-interface model potentially admits trapped numerical waves that reflect back and forth repeatedly from one interface to another. If the reflections cause amplification, this can lead to unbounded growth of numerical solutions. The factors that control this are: magnitude of the reflection coefficients, which is the source of growth; dissipation of waves as they travel between interfaces, which is a source of attenuation; and travel time between interfaces, which determines how frequently any reflection circuit that causes growth is repeated. All of the arguments of this paper consist of working out consequences of various combinations of these factors that may be of practical interest.

In particular, we investigate two kinds of stability. First, "stability" or "LaxRichtmyer stability" refers to the usual Lax-Richtmyer definition for time-dependent finite-difference models, or to variants thereof such as "GKS-stability" (Definition 3.3 in the well-known paper by Gustafsson, Kreiss, and Sundström [10]). A difference model that is stable in this sense may admit solutions that grow with time, provided that the growth does not get worse as the mesh is refined. This is what is needed to ensure convergence as the mesh size approaches zero to the correct solution of the time-dependent differential equation, for each fixed time $t$. On the other hand, to be " $P$-stable" [1], a model must admit no growing solutions at all. (See Section 3 for the precise definition. Such a model is also sometimes called "time-stable" [29].) Although the theory here is not as well developed, such a condition is needed if a time-dependent difference model is to be used to obtain steady-state solutions, as is common in computational aerodynamics. As a rule of thumb, we will show that $P$-instability is very often associated with reflection coefficients greater than 1 in magnitude, and Lax-Richtmyer instability with reflection coefficients that are infinite.

Section 1 reviews stability theory for one-boundary difference models (Proposition 1). Section 2 investigates interfaces separated by a fixed number of grid points $\Delta j$ as the mesh is refined, as in the fourth-order boundary condition mentioned above. Here the travel times go to zero with the mesh spacing, with the effect that finite reflection coefficients greater than 1 can cause catastrophic unstable growth (Propositions 3,4, 4'). Conversely, reflection coefficients smaller than 1 ensure stability (Proposition 5). Section 3 considers interfaces separated by a fixed distance $\Delta x$ as the mesh is refined, as in the problem on $[0,1]$ mentioned above. Here, the travel times are independent of mesh spacing, so large finite reflection coefficients can cause $P$-instability (Proposition 7), but not Lax-Richtmyer instability (Proposition 6 ). In this context multiple reflections may convert the weak instability of a single interface to a catastrophic instability (Proposition 8), but only if the unstable interface is of the sort with an infinite reflection coefficient (Proposition 9). Once again, reflection coefficients smaller than 1 in norm ensure stability (Proposition 10), 
and if the model is dissipative, it suffices to look at the reflection coefficients for the partial differential equation rather than its numerical approximation (Proposition 11).

For convenience of reference, here is a list of the explicit examples presented here to illustrate various points. The symbol $A$ indicates the modulus of a reflection coefficient, and $S_{n}$ the solution norm at time step $n$. These quantities will be made more precise later on.

1. Algebraically unstable one-boundary model (one boundary, $A=\infty, S_{n} \sim$ const $n$ ).

2. Exponentially unstable concatenation of three stable dissipative formulas (fixed- $\Delta j, A>1, S_{n} \sim$ const $^{n}$ ).

3. $P$-stability guaranteed by reflection coefficients less than 1 (fixed- $\Delta j$ or $\Delta x, A$ $\leqslant 1, S_{n} \sim$ const).

4. $P$-instability caused by reflection coefficients greater than 1 (fixed- $\Delta x, A>$ $1, S_{n} \sim$ const $\left.^{t}\right)$.

5. Exponential instability caused by interaction of two algebraically unstable boundaries (fixed $-\Delta x, A=\infty, S_{n} \sim(\Delta j)^{\text {const } t}$ ).

The reader may be disappointed at the artificiality of some of these examples, especially (2) and (3), and he may wonder how helpful wave reflection ideas can be in practice for the design of difference schemes. A full answer to this question will have to await further experience. Nevertheless, there is no doubt that the instability mechanisms described here are real and deserve to be understood. At present, virtually no difference models containing multiple interfaces have been shown to be stable. Perhaps the ideas here, such as Proposition 5, can help bring about a change in this situation.

Much of the material in this paper can be found in Section 6 of the author's Ph. D. dissertation [24]. For some numerical illustrations, see [28]. For a different analysis of stability for two-boundary problems that is closely related to the present one, see the report [8] by Giles and Thompkins, which is mainly concerned with $P$-stability. Giles and Thompkins consider parasitic wave propagation for models with variable as well as constant coefficients.

The phenomenon of instability caused by trapped wave packets can also occur in two-dimensional problems when the domain contains a corner. Osher has given examples of hyperbolic systems (not difference models) in corners that are ill-posed because of trapped waves [19], while Sarason and Smoller have shown that for a $2 \times 2$ strictly hyperbolic system such as the second-order wave equation, this cannot happen [21]. But trapped numerical waves may render a finite-difference model of even the latter sort unstable. The principles involved are precisely those of this paper, but we will discuss corners elsewhere.

The reader interested in getting to the main ideas quickly may find it possible to turn directly to Section 2 .

1. Review of Wave Propagation and Stability for One-Boundary Difference Models. Consider a linear first-order hyperbolic system of partial differential equations

$$
u_{t}=A u_{x}
$$


with initial data

$$
u(x, 0)=f(x) .
$$

Here $u(x, t)$ and $f(x)$ are $N$-vectors, $A$ is a constant $N \times N$ matrix, and the spatial domain is $\mathbf{R}$. The statement that (1.1) is hyperbolic means that $A$ has real eigenvalues $\left\{\mu_{\nu}\right\}, 1 \leqslant \nu \leqslant N$, and a complete set of associated eigenvectors $\left\{U_{\nu}\right\}$. It follows that if $\xi \in \mathbf{R}$ is an arbitrary wave number, then (1.1) admits $N$ linearly independent solutions of the form $u(x, t)=U \exp (i(\omega t+\xi x))$, namely the waves

$$
u(x, t)=U_{\nu} e^{i\left(\omega_{\nu}(\xi) t+\xi x\right)}
$$

with $\omega_{\nu}(\xi)=\mu_{\nu} \xi$. $\omega_{\nu}$ is called the frequency of (1.3), and the $N$-valued linear function $\omega=\omega(\xi)$ is the dispersion relation for (1.1). Each wave (1.3) propagates uniformly with no change in shape at the velocity $-\mu_{\nu}$, hence leftward or rightward depending on whether $\mu_{\nu}$ is positive or negative, respectively.

Since the vectors $U_{\nu}$ span $\mathbf{R}^{N}$, any $f \in L^{2}\left(\mathbf{R}^{N}\right)$ can be written as a Fourier integral with respect to $\xi$ of waves (1.3). It follows, by Parseval's theorem, that $\|u(\cdot, t)\|$ is constant with respect to $t$; a fortiori, for any fixed $t$ one has

$$
\|u(\cdot, t)\| \leqslant \text { const }\|f\|,
$$

which is to say, (1.1)-(1.2) is well-posed in $L^{2}$. Related well-posedness bounds continue to hold under reasonable assumptions if (1.1) is given a zeroth-order term $B u$, an inhomogeneous term $F(x, t)$, or variable coefficients, although in these circumstances some growth at a bounded rate in $t$ must be permitted. For simplicity, we will ignore these possibilities.

Let $u$ be approximated by a vector grid function $v_{j}^{n}=v(j h, n k) \approx u(j h, n k)$, where $k$ is the time step and $h$ is the space step. $\left\{v_{j}^{n}\right\}$ will be determined iteratively as the solution of an $s+2$-level finite-difference formula

$$
Q_{-1} v^{n+1}=\sum_{\sigma=0}^{s} Q_{\sigma} v^{n-\sigma},
$$

where each $Q_{\sigma}$ is a spatial finite-difference operator with constant matrix coefficients of dimension $N \times N$. Let $Q$ be a name for (1.5). As with the differential equation, one can show that $Q$ admits solutions

$$
v_{j}^{n}=V e^{i(\omega t+\xi x)}, \quad x=j h, t=n k, V \in \mathbf{R}^{N} .
$$

For each $\xi \in \mathbf{R}$, in fact it permits in general not $N$ but $(s+1) N$ distinct values of $\omega$, whose relation to $\xi$ constitutes the dispersion relation for (1.5). These values depend nonlinearly on $\xi$, and they are not necessarily real. A solution with $\xi \in \mathbf{R}$ and $\operatorname{Im} \omega>0$ decays with $t$, but a solution with $\xi \in \mathbf{R}$ and $\operatorname{Im} \omega<0$ grows at the rate $e^{|\operatorname{Im} \omega| t}=$ const $^{n}$, and if $Q$ admits a solution of this kind, it is unstable. On the other hand, if there are no such growing modes, and if any modes with $\xi, \omega \in \mathbf{R}$ are nondefective in a sense we will not go into, then $Q$ is stable. Thus, stability for a constant coefficient finite-difference model on an unbounded domain can be investigated by a fairly straightforward process of Fourier analysis. For details, see [20].

Let $Q$ be stable and admit a solution (1.6) with $\xi, \omega \in \mathbf{R}$. It can be shown that the dispersion relation for (1.5) determines a function $\tilde{\omega}=\tilde{\omega}(\tilde{\xi})$ for $\tilde{\xi}, \tilde{\omega}$ in a neighborhood of $\xi, \omega[27$, Lemma 3.2], and that the energy associated with the wave (1.6) 
propagates at the group velocity

$$
C(\xi, \omega)=-\frac{d \tilde{\omega}}{d \tilde{\xi}}(\xi, \omega)
$$

The precise meaning of this statement is asymptotic: if $Q$ is given initial data

$$
f_{j}^{\sigma}=\phi(x-C t) V e^{i(\omega t+\xi x)}, \quad 0 \leqslant \sigma \leqslant s, t=\sigma k, x=j h,
$$

for some smooth function $\phi$, then the solution at a later time will be

$$
v_{j}^{n} \approx \phi(x-C t) V e^{i(\omega t+\xi x)}
$$

with the approximation becoming better as $\phi$ is made smoother. See, for example, Lemma 5.1 of [27].

Example 1. As an example, consider the leap frog (LF) model

$$
v_{j}^{n+1}=v_{j}^{n-1}+\lambda\left(v_{j+1}^{n}-v_{j-1}^{n}\right), \quad \lambda=\frac{k}{h}=\text { const },
$$

of $u_{t}=u_{x}$. By inserting $v(x, t)=e^{i(\omega t+\xi x)}$, one finds that the dispersion relation is

$$
\sin \omega k=\lambda \sin \xi h \text {. }
$$

Differentiation leads to the group velocity formula

$$
C(\xi, \omega)=-\frac{\cos \xi h}{\cos \omega k} \text {. }
$$

Thus a well-resolved wave, i.e., one with $\xi h, \omega k \approx 0$, propagates under LF with group velocity $C \approx-1$. On the other hand,LF also admits many waves with $\xi h$ or $\omega k$ not small. The extreme cases are the "parasitic" solutions $(\xi, \omega)=(\pi / h, 0),(0, \pi / k)$, and $(\pi / h, \pi / k)$, which by (1.10) have group velocities $+1,+1$, and -1 , respectively. For the first two of these, the sign of $C$ reveals that energy propagates in the physically wrong direction. In fact, for each sufficiently small frequency $\omega \in \mathbf{R}$, (1.9) gives two distinct wave numbers $\xi$ in the fundamental range $[-\pi / h, \pi / h]$, and by (1.10), one of the corresponding waves propagates leftwards and the other propagates rightwards. See [25] or [30] for illustrations.

Returning to the general model $Q$ of (1.5), let us change the notation and rewrite (1.6) in the more convenient form

$$
v_{j}^{n}=V \kappa^{j} z^{n}, \quad \kappa, z \in \mathbf{C}-\{0\},
$$

where $\kappa=e^{i \xi h}$ and $z=e^{i \omega k}$. (For full generality one must permit a further multiplicative factor $j^{\delta}$ to represent certain defective modes. Such modes are rarely important in practice, however, so in all of what follows we assume $\delta=0$, although the results remain valid in the general situation. The reader is referred to [27] for more complete details.) A solution (1.11) with $|\kappa|=|z|=1$ and $C \leqslant 0$ (resp. $\geqslant 0$ ) will be called leftgoing (resp. rightgoing). For obvious geometric reasons it also makes sense to say that a solution with $|z| \geqslant 1$ is leftgoing if $|\kappa|>1$ and rightgoing if $|\kappa|<1$. It can be shown under reasonable assumptions (see [10]) that for any $z$ with $|z| \geqslant 1, Q$ admits a family of $R=N l$ linearly independent rightgoing and $L=N r$ linearly independent leftgoing solutions (1.11), where $l$ and $r$ denote the numbers of grid points to the left and right of center, respectively, covered by the stencil of $Q$ [27, Section 3]. Therefore, the general solution to (1.5) of the form $v_{j}^{n}=z^{n} \phi_{j}$ is a linear combination

$$
v_{j}^{n}=z^{n} \sum_{m=1}^{R+I} \alpha_{m} V_{m} \kappa_{m}^{j} .
$$


If we relabel $\alpha, V$, and $\kappa$ by $\beta, W$, and $\mu$ for leftgoing components, this becomes

$$
\begin{gathered}
v_{j}^{n}=z^{n} \sum_{m=1}^{R} \alpha_{m} V_{m} \kappa_{m}^{j}+z^{n} \sum_{m=1}^{l} \beta_{m} W_{m} \mu_{m}^{j} . \\
(\text { RIGHTGOING) }
\end{gathered}
$$

We emphasize that the leftgoing and rightgoing waves in this sum have very little to do with the waves admitted by the original equation (1.1).

Let a left-hand boundary be introduced at $x=0$, so that the spatial domain becomes $\mathbf{R}^{+}$and $j$ is restricted to $j \geqslant 0$. Now (1.1) must be supplemented by as many additional scalar boundary conditions as there are inflowing characteristics at $x=0$, and if this is done in the natural way, well-posedness is guaranteed [13]. But we pass over these details and consider the finite-difference model. Regardless of the characteristics of (1.1), (1.5) will have to be supplemented by $R=N l$ additional boundary conditions, one for each rightgoing numerical solution component. For simplicity, we take these to be homogeneous and of the form

$$
v_{j}^{n+1}=\sum_{\sigma=-1}^{M_{1}} \sum_{i=0}^{M_{2}} \gamma_{i \sigma} v_{i}^{n-\sigma}, \quad 0 \leqslant j \leqslant l-1,
$$

for some integers $M_{1}$ and $M_{2}$ and $N \times N$ matrices $\gamma_{i \sigma}$. Let $\bar{Q}$ be a name for the initial-boundary value problem model (1.5), (1.13).

In practice, it can easily happen that $\bar{Q}$ is unstable. A theory of such instability was developed a decade ago by Kreiss, Osher, and others, and described at length in the well-known paper [10] by Gustafsson, Kreiss, and Sundström-henceforth "GKS". In [26] and [27] the Kreiss/Osher theory has been given the following interpretation. If $|z| \geqslant 1$ is fixed, then the general superposition (1.12) of leftgoing and rightgoing waves does not satisfy (1.13), and hence is not a solution to $\bar{Q}$. Instead, (1.13) can be thought of as a set of $R=N l$ reflection conditions relating rightgoing to leftgoing waves at the boundary. These conditions are obtained by substituting (1.12) in (1.13) and then collecting terms in $\alpha_{m}$ and $\beta_{m}$, so that one gets

$$
E(z)\left[\begin{array}{c}
\alpha_{1} \\
\vdots \\
\alpha_{R}
\end{array}\right]=D(z)\left[\begin{array}{c}
\beta_{1} \\
\vdots \\
\beta_{L}
\end{array}\right]
$$

for some $R \times R$ matrix $E(z)$ and $R \times L$ matrix $D(z)$. For most $z, E(z)$ will be nonsingular, and (1.14) determines the reflected wave coefficients as a bounded function of the incident ones. If we write $A=E^{-1} D$, so that $A(z)$ is the $R \times L$ reflection matrix for the given boundary conditions, then this function has the form

$$
\alpha=A(z) \beta=[E(z)]^{-1} D(z) \beta .
$$

(This $A(z)$ has nothing to do with the coefficient matrix of (1.1).) However, it may happen that for some $\left|z_{0}\right| \geqslant 1, E\left(z_{0}\right)$ is singular, and in this case (1.14) permits a solution consisting of rightgoing waves in the absence of leftgoing waves. This will cause instability. If in this situation $A(z)$ is unbounded as $z \rightarrow z_{0}$, then an infinitereflection coefficient exists at $z_{0}$, and the instability will be particularly severe [27]. 
Thus the Kreiss/Osher theory leads to the following "GKS stability theorem":

Proposition 1 [10], [27]. The initial-boundary value problem model $\bar{Q}$ is GKS-unstable if and only if $E(z)$ is singular for some $|z| \geqslant 1$. Equivalently, it is GKS-unstable if and only if for some $|z| \geqslant 1$ it admits a nonzero solution $v_{j}^{n}=z^{n} \phi_{j}(1.12)$ consisting entirely of rightgoing wave components.

Proof. See [27] for a precise statement and proof.

The notion of "GKS-stability" employed in this result is a fairly complicated one given as Definition 3.3 in [10]. See [27] for a discussion of its meaning. For the remainder of this paper "stable" means "GKS-stable", except where otherwise stated.

Example 1, continued. To return to the previous example, suppose LF is applied on $x \geqslant 0$ with the numerical boundary condition

$$
v_{0}^{n+1}=v_{1}^{n+1} .
$$

In $\kappa, z$ notation, the dispersion relation (1.9) and group velocity (1.10) for LF are

$$
z-\frac{1}{z}=\lambda\left(\kappa-\frac{1}{\kappa}\right), \quad C=-\frac{\kappa+1 / \kappa}{z+1 / z},
$$

and (1.16) imposes the additional condition $\kappa=1$. One sees immediately that the wave $(\kappa, z)=(1,-1)$, i.e., $v_{j}^{n}=(-1)^{n}$, satisfies both the interior formula and the boundary condition and has $C>0$. Therefore, by Proposition 1, the model (1.8), (1.16) is unstable. By contrast, the condition $v_{0}^{n+1}=v_{1}^{n}$ is satisfied by no rightgoing solutions to LF, so with this boundary condition LF would be stable.

This example is one of those with an infinite reflection coefficient. To see this, note that for each $|z| \geqslant 1$, (1.17) gives two values of $\kappa$ related by $\kappa_{2}=-1 / \kappa_{1}$. Let these be denoted by $\kappa$ and $\mu$, where $\kappa$ is the "rightgoing" value with $\operatorname{Re} \kappa \operatorname{Re} z \leqslant 0$ and $|\kappa| \leqslant 1$, for which $C \geqslant 0$ if $|\kappa|=1$, and $\mu$ is the "leftgoing" one with $\operatorname{Re} \kappa \operatorname{Re} z \geqslant 0$ and $|\kappa| \geqslant 1$. Then for this problem the superposition (1.12) takes the form $v_{j}^{n}=\alpha \kappa^{j}$ $+\beta \mu^{j}$. To calculate the reflection coefficient we substitute this in (1.16) and obtain $\alpha+\beta=\alpha \kappa+\beta \mu$, that is, $\alpha=A \beta$ with

$$
A(z)=-\frac{1-\mu}{1-\kappa}
$$

This quotient becomes infinite when $z=-1, \kappa=1, \mu=-1$.

The unstable behavior of this difference model is illustrated in Figures 4.1-4.2 and Figures 5.1-5.4 of [24] and in Figures 3, 4 of [27].

2. Two Interfaces Separated By a Fixed Number of Grid Points $\Delta j$. The stability result of Proposition 1 is illustrated in Figure 1. If a set of numerical waves reflects at a boundary with a gain in amplitude, as in Figure 1a, this does not constitute instability. It may force the constant in a discrete estimate analogous to (1.4) to be large, but it does not preclude the existence of such an estimate. On the other hand, if the boundary can produce radiated energy in the presence of no incident energy at all, as in Figure 1b, then it is unstable. 


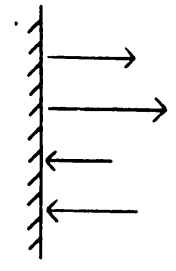

(a) stable

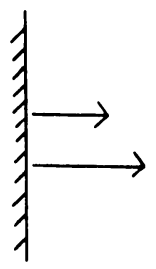

(b) unstable

\section{FIGURE 1}

Stable and unstable solutions $z^{n} \phi_{j}$ at a left-hand boundary

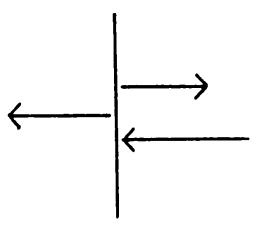

(a) stable

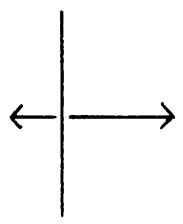

(b) unstable

FIGURE 2

Stable and unstable solutions $z^{n} \phi_{j}$ at an internal interface

Suppose now that $\bar{Q}$ is a model containing not a boundary but an internal interface of some kind separating two difference schemes $Q_{\text {- }}$ and $Q_{+}$(possibly identical). The interface might be a complicated structure extending over several grid points, or it might be simply an abrupt change of coefficient, of difference formula, or of mesh size. It is plausible that the picture should change to that of Figure $2: \bar{Q}$ is unstable if and only if it permits a solution $z^{n} \phi_{j}$ that is outgoing from the interface on both sides. This conclusion can be derived rigorously from Proposition 1 by folding the interface problem into an initial-boundary value problem for a system of equations of twice the original size [3], [5], [6], [16], [24].

Reflection equations for an internal interface analogous to (1.14)-(1.15) for a boundary can be obtained by the same folding idea. For each $|z| \geqslant 1$, there are $R^{-}+L^{+}$linearly independent waves that may be incident at the interface from both sides, and $L^{-}+R^{+}$that may be radiated. The full reflection equation is the linear system describing how the coefficients of these wave components are related,

$$
E(z)\left[\begin{array}{c}
\beta_{1}^{-} \\
\vdots \\
\beta_{L^{-}}^{-} \\
\alpha_{1}^{+} \\
\vdots \\
\alpha_{R^{+}}^{+}
\end{array}\right]=D(z)\left[\begin{array}{c}
\alpha_{1}^{-} \\
\vdots \\
\alpha_{R^{-}}^{-} \\
\beta_{1}^{+} \\
\vdots \\
\beta_{L^{+}}^{+}
\end{array}\right],
$$

where $E$ and $D$ are matrix functions of dimensions $\left(L^{-}+R^{+}\right) \times\left(L^{-}+R^{+}\right)$and $\left(L^{-}+R^{+}\right) \times\left(R^{-}+L^{+}\right)$(cf. (1.14)). However, in this paper we will only need the response of an interface on one side to a wave incident on that side. The corresponding reflection equation is the projection of (2.1) onto a one-sided domain and 
range. In the case of incidence on the right, for example, it has the form

$$
E(z)\left[\begin{array}{c}
\alpha_{1}^{+} \\
\vdots \\
\alpha_{R^{+}}^{+}
\end{array}\right]=D(z)\left[\begin{array}{c}
\beta_{1}^{+} \\
\vdots \\
\beta_{L^{+}}^{+}
\end{array}\right]
$$

where $E$ is $R^{+} \times R^{+}$and $D$ is $R^{+} \times L^{+}$. When $E(z)$ is nonsingular, (2.2) can be solved to yield and equation analogous to (1.15),

$$
\alpha^{+}=A(z) \beta^{+}
$$

where $A$ is $R^{+} \times L^{+}$. Note that although wave modes on the left of the interface do not appear in (2.3), the projection process by which this equation is obtained imposes the condition that the wave energy on the left is nonzero in the leftgoing components only. In other words (2.2)-(2.3) describes the response of the interface to energy incident on the right.

Now consider a finite-difference model $\bar{Q}$ with $p$ interfaces located at grid points $j=j_{1}, \ldots, j_{p}$, and write $\Delta j=j_{p}-j_{1}$. (To be precise, each $j_{\nu}$ is a half-integer, with one difference formula applied on $j_{\nu-1}<j<j_{\nu}$ and another on $j_{\nu}<j<j_{\nu+1}, j \in \mathbf{Z}$.) In this section, the indices $j_{\nu}$ are to be kept fixed as $h, k \rightarrow 0$, and we recognize this assumption by calling $\bar{Q}$ a model of "fixed- $\Delta j$ " type. As mentioned in the Introduction, a fixed $-\Delta j$ problem might come up in the analysis of adaptive mesh-refinement procedures, or with any boundary or interface discretization that involves more than two distinct difference formulas. We obtain the following stability criterion:

Proposition 2. A fixed- $\Delta j$ multi-interface finite-difference model is unstable if and only if for some $|z| \geqslant 1$ it admits a nonzero solution $z^{n} \phi_{j}$ containing only leftgoing waves to the left and rightgoing waves to the right of all interfaces.

Proof. The situation is illustrated in Figure 3. For a proof, one can relabel the grid points so that the interval from $j_{1}$ to $j_{p}$ becomes one complicated interface separating the two regions $j<j_{1}$ and $j>j_{p}$. Then the folding argument mentioned above for a single interface applies.

Remark. In the case of an initial-boundary value problem with a boundary at the left, say, the region to the left of the interfaces in Proposition 2 becomes finite in extent (or possibly empty, depending on labeling), so in principle one should not restrict the search for unstable modes to solutions that are leftgoing there. But in this region the difference formula is necessarily one-sided, which implies, under the usual assumptions, that it admits leftgoing waves only for $|z| \geqslant 1$. Therefore the change is vacuous.

From the wave propagation point of view the following result should now be unsurprising.

Proposition 3. For the stability of a fixed- $\Delta j$ multi-interface model, it is not sufficient that the individual interfaces be stable.

Remark. Stability of the individual interfaces is presumably not necessary, either. 


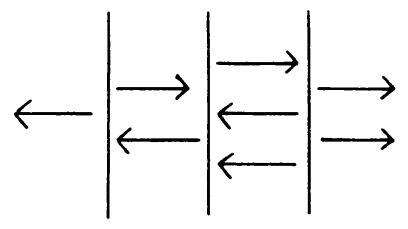

(a) interior

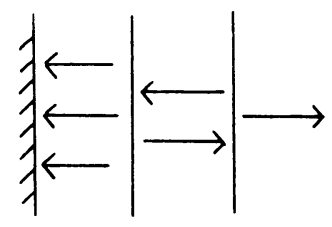

(b) boundary

FIGURE 3

Unstable multi-interface solutions $z^{n} \phi_{j}$ at

an interior interface and at a boundary

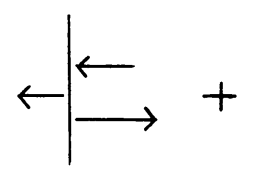

(a)

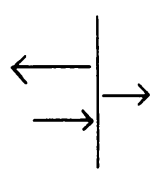

ב

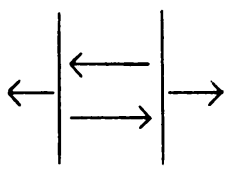

(b)

FIGURE 4

The concatenation of stable interfaces may be unstable

Proof. The proof consists of exhibiting Example 2, below, but the idea behind it is indicated in Figure 4. Imagine two interfaces at which waves can reflect with a reflection coefficient greater than 1 . When these are placed together, it might happen that the reflected wave from each interface serves to stimulate the reflected wave from the other. A process of reflection back and forth will then ensue in which at each circuit, the amplitude grows by a factor const $>1$. Since one circuit takes only a fixed number of time steps, this process will cause growth at a rate $\left\|v^{n}\right\|=$ const $^{n}$, which is an explosive instability.

Example 2. Let $u_{t}=u_{x}$ on $x \geqslant 0$ be modeled by an "interior" formula for $j \geqslant 2$ combined with additional boundary formulas at $j=0$ and $j=1$. The interior formula is an upstream difference with some added dissipation:

$$
v_{j}^{n+1}=v_{j}^{n}+\lambda\left(v_{j+1}^{n}-v_{j}^{n}\right)+\frac{9 \lambda}{8}\left(v_{j+1}^{n}-2 v_{j}^{n}+v_{j-1}^{n}\right), \quad j \geqslant 2 .
$$

The formula at $j=0$ is a linear combination of upstream differences:

$$
v_{0}^{n+1}=v_{0}^{n}+\frac{\lambda}{8}\left(\frac{v_{2}^{n}-v_{0}^{n}}{2}\right)+\frac{7 \lambda}{8}\left(\frac{v_{3}^{n}-v_{0}^{n}}{3}\right) .
$$

At $j=1$ we use a leapfrog formula with some added dissipation:

$$
v_{1}^{n+1}=v_{1}^{n-1}+\lambda\left(v_{2}^{n}-v_{0}^{n}\right)+\varepsilon\left(v_{2}^{n+1}-2 v_{1}^{n+1}+v_{0}^{n+1}\right) .
$$

It is verified in Section 6.3 of [24] that if $\lambda=\frac{1}{8}$ and $\varepsilon=1036 / 83205$, then (2.4)-(2.6) is exponentially unstable, admitting a solution $v_{j}^{n}=z^{n} \phi_{j}$ with $z=129 / 128$. The eigensolution $\phi$ has the form $\left(\frac{1}{4}, 1, \frac{1}{2}, \frac{1}{4}, \frac{1}{8}, \ldots\right)$, and can be viewed as the superposition of leftgoing and rightgoing waves represented in Figure $3 \mathrm{~b}$. A numerical experiment confirms that $(2.4)-(2.6)$ is highly unstable and blows up like $(129 / 128)^{n}$ [24]. 
We have chosen such an unwieldy example because it is contrived to have a special additional property: all of the formulas (2.4)-(2.6) are dissipative. This is of interest because, as a matter of practical experience, dissipativity often ensures stability. For the case of a single interface, it has been proved by Ciment [6] (for interfaces) and later by Goldberg and Tadmor [9] (for boundaries) that under reasonable hypotheses, this is always true. See also Section 6.2 of [24]. Later, it was claimed by Oliger [17] that the same must hold with multiple interfaces. However, the example above shows this is not so. We formulate this conclusion as a new proposition:

Proposition 4. In a fixed- $\Delta j$ model with two or more interfaces, such as an initial-boundary value problem model with distinct boundary conditions at $j=0$ and $j=1$, dissipativity of each individual difference formula is not sufficient to ensure stability.

It would, of course, be more satisfying to find an illustration of this principle that was somewhat realistic.

Example 2 also serves to illustrate another (weaker) stability principle. In some circles, where the Kreiss/Osher theory is considered too complicated for practical work, the "von Neumann" or "Fourier method" for heuristic stability analysis is used instead. This idea, proposed by Trapp and Ramshaw [23] (not by von Neumann), is to check the numerical boundary formulas for amplification factors greater than 1 just as if they were interior formulas, and hope that if there are none such, the model will be stable. In general, there is little reason to expect this procedure to work, and indeed the heuristic justification of it by Trapp and Ramshaw is not really valid. Yet because of the algebraic simplicity of the difference formulas usually encountered, the idea is surprisingly reliable in practice [22]. In particular, for a dissipative difference model with a solvable boundary condition applied at a single point, it can readily be shown that the Fourier condition is sufficient for stability [9].

But Example 2 confirms that the same does not hold when there is more than one boundary condition:

Proposition 4'. In an initial-boundary value problem model involving distinct boundary conditions at $j=0$ and $j=1$, the "von Neumann method" of boundary condition analysis is not sufficient to ensure stability.

If the stability of each interface individually is not enough for a general stability test, what is? The unfortunate answer is that for a complete analysis one must investigate all possible modes $z^{n} \phi_{j}$ suggested by Propositions 1 or 2 to see if they satisfy the boundary conditions. The difficulty with this computation is that its size grows with the total width of the interface region: one must study a matrix function $E(z)$ of dimension approximately $\Delta j$ in the scalar case, $N \Delta j$ in general. The required investigation can be prohibitively difficult.

However, various sufficient but not necessary conditions for stability can be derived that involve the interfaces individually. Consider the two-interface model $\bar{Q}=Q_{-}\left|Q_{0}\right| Q_{+}$illustrated in Figure 5. Here $Q_{-}, Q_{0}$, and $Q_{+}$are constant-coefficient difference formulas with stencil parameters $\left\{l_{-}, r_{-}\right\},\{l, r\},\left\{l_{+}, r_{+}\right\}$, and $j_{1}$ and $j_{2}$ are 


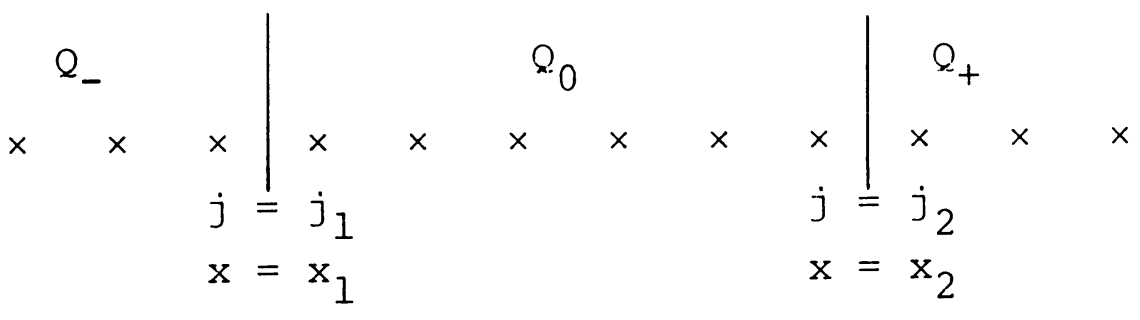

FIGURE 5

Two-interface model

half-integers with $\Delta j=j_{2}-j_{1} \geqslant 1$. The interface at $j_{1}$ consists simply of an abrupt change from $Q_{-}$applied for $j<j_{1}$ to $Q_{0}$ applied for $j>j_{1}$, and similarly at $j_{2}$. We assume that each interface $Q_{-} \mid Q_{0}$ and $Q_{0} \mid Q_{+}$is individually stable, and seek a condition to ensure that no unstable solution $z^{n} \phi_{j}$ of $\bar{Q}$ with $|z| \geqslant 1$ (as in Figure 4b) can exist. To ensure decoupling of $Q_{-}$and $Q_{+}$, we assume further $r_{-} \leqslant r+\Delta j$ and $l_{+} \leqslant l+\Delta j$.

Let $Q_{0}$ admit $R$ rightgoing and $L$ leftgoing solutions, labeled as in (1.12). Let $K(z)$ and $M(z)$ be the $R \times R$ and $L \times L$ nonsingular matrices

$$
K(z)=\operatorname{diag}\left(\kappa_{1}, \ldots, \kappa_{R}\right), \quad M(z)=\operatorname{diag}\left(\mu_{1}, \ldots, \mu_{L}\right),
$$

and let $V$ and $W$ be the $N \times R$ and $N \times L$ matrices with columns $V_{m}$ and $W_{m}$,

$$
V(z)=\left(V_{1}, \ldots, V_{R}\right), \quad W(z)=\left(W_{1}, \ldots, W_{L}\right) .
$$

Then (1.12) can be rewritten

$$
v_{j}^{n}=z^{n}\left[V(z) K(z)^{j} \alpha+W(z) M(z)^{j} \beta\right] .
$$

By definition of $V, K, W$, and $M$, this expression satisfies $Q_{0}$ for all $j$, regardless of $\alpha$ and $\beta$. Conversely, a function $v_{j}^{n}=z^{n} \phi_{j}$ satisfies $Q_{0}$ for $j_{1}<j<j_{2}$ only if it has a representation (2.7) valid in $j_{1}-l<j<j_{2}+r$ for some $\alpha$ and $\beta$. The question is, for which $\alpha$ and $\beta$, if any, can a function $v_{j}^{n}$ defined by (2.7) in $j_{1}-l<j<j_{2}+r$ be extended to a solution of $\bar{Q}$ for all $j$ that is leftgoing in $j<j_{1}$ and rightgoing in $j>j_{2}$ ? The answer is: for precisely those $\alpha, \beta$ satisfying the reflection equations

$$
\alpha=A_{1} \beta, \quad \beta=A_{2} \alpha,
$$

where $A_{1}$ is an $R \times L$ matrix as in (2.3) relating $\alpha$ to $\beta$ at the $Q_{-} \mid Q_{0}$ interface, and $A_{2}$ is an $L \times R$ matrix relating $\beta$ to $\alpha$ at $Q_{0} \mid Q_{+}$. This follows from the construction of (2.2). The assumption that each interface is stable in isolation has permitted us to pass from the form (2.2) to (2.3), since it implies by Proposition 1 that $E_{1}(z)$ and $E_{2}(z)$ are nonsingular for each $|z| \geqslant 1$.

The matrix $A(z)$ of (1.15) was effectively defined with respect to the grid point $j=0$, in the sense that it is at that point where a solution (1.12) to $\bar{Q}$ has the form $V \alpha+W \beta$ with $\alpha=A(z) \beta$. For the present problem, it is more natural for $A_{1}$ to be defined with respect to the grid point $j_{1}$, and $A_{2}$ with respect to $j_{2}$. We can accomplish this by replacing $A_{1}$ in (2.8) by $K^{-j_{1}} A_{1} M^{j_{1}}$ and $A_{2}$ by $M^{-j_{2}} A_{2} K^{j_{2}}$. Equation (2.8) becomes

$$
K(z)^{j_{1}} \alpha=A_{1}(z) M(z)^{j_{1}} \beta
$$


and

$$
M(z)^{j_{2}} \beta=A_{2}(z) K(z)^{j_{2}} \alpha .
$$

With this somewhat cumbersome notation it is possible to state a simple lemma on the existence of solutions $v_{j}^{n}=z^{n} \phi_{j}$ to $\bar{Q}$.

LEMMA 1. The fixed- $\Delta j$ two-interface model $\bar{Q}$ described above admits a solution $v_{j}^{n}=z^{n} \phi_{j}$ with $|z| \geqslant 1$ consisting of outgoing waves only in $j\left\langle j_{1}\right.$ and $j>j_{2}$ if and only if the $L \times L$ matrix

$$
E_{L}(z)=M(z)^{-\Delta j} A_{2}(z) K(z)^{\Delta j} A_{1}(z)
$$

has an eigenvalue 1.

Proof. Suppose $\bar{Q}$ has a solution $v_{j}^{n}=z^{n} \phi_{j}$ of the kind described. Let $\alpha$ and $\beta$ be the coefficient vectors for the representation (2.7) of $v$ in $j_{1}-l<j<j_{2}+r$. By definition of $A_{1}$ and $A_{2}$, the equations (2.9) and (2.10) must hold. Multiplying them together gives

$$
M(z)^{j_{2}} \beta=A_{2}(z) K(z)^{\Delta_{j}} A_{1}(z) M(z)^{j_{1}} \beta,
$$

that is,

$$
\left[M(z)^{j_{1}} \beta\right]=E_{L}(z)\left[M(z)^{j_{1}} \beta\right] .
$$

Thus $M(z)^{j_{1}} \beta$ is an eigenvector of the sort required.

Conversely, if $E_{L}(z)$ has an eigenvalue 1 , let $\beta$ be $M(z)^{-j_{1}}$ times a corresponding eigenvector, and define $\alpha$ by (2.9). Then by definition of $\beta,(2.10)$ is satisfied also, so $\bar{Q}$ has a solution of the required kind.

Lemma 1 now makes it possible to give sufficient conditions for stability based on $A_{1}$ and $A_{2}$ alone.

PROPOSITION 5. In the fixed- $\Delta j$ two-interface problem described above, in which each interface individually is stable, a sufficient condition for stability is

$$
\left\|A_{1}(z)\right\|\left\|A_{2}(z)\right\|<1 \text { for all }|z| \geqslant 1
$$

in any norm $\|\cdot\|$ subordinate to a vector norm.

Remark. $A_{1}$ and $A_{2}$ are rectangular matrices, i.e., operators $A_{1}: \mathrm{C}^{L} \rightarrow \mathrm{C}^{R}$ and $A_{2}$ : $\mathbf{C}^{R} \rightarrow \mathbf{C}^{L}$. The norms in Proposition 5 are the operator norms subordinate to any norms on $\mathbf{C}^{L}$ and $\mathbf{C}^{R}$, which must, however, be the same for both $A_{1}$ and $A_{2}$.

Proof. By the definitions of rightgoing and leftgoing we have $\left|\kappa_{m}\right| \leqslant 1 \leqslant\left|\mu_{m}\right|$ for all $z$ and $m$, hence $\|K(z)\|,\left\|M(z)^{-1}\right\| \leqslant 1$ in any norm. Together with the hypotheses and (2.11) this implies $\left\|E_{L}(z)\right\|<1$ for each $|z| \geqslant 1$, which precludes the existence of the eigenvalue 1 of Lemma 1.

Example 3. Here we reproduce a " $P$-stability" result of Beam, Warming and Yee [1] by considering reflection coefficients. Let $u_{t}=u_{x}$ on [0,1] be modeled by any of the " $A$-stable" formulas $Q$ of Beam and Warming, which consist of the usual three-point difference operator in $x$ coupled with an $A$-stable linear multistep formula in $t$. Examples are the backward Euler and trapezoidal (= Crank-Nicolson) formulas. Let the boundary conditions be $v^{n+1}=0$ at $x=1, j=\Delta j+1 \geqslant 2$, and $q$ th-order space extrapolation $(q \leqslant \Delta j+1)$

$$
(K-1)^{q} v_{0}^{n+1}=0
$$


at $x=j=0$, where $K$ denotes the shift operator $K v_{j}^{n}=v_{j+1}^{n}$. We claim that for any fixed $\Delta j, \bar{Q}$ admits no solution $v_{j}^{n}=z^{n} \phi_{j}$ with $|z|>1$.

Since the spatial difference in $Q$ is just $\left(K-K^{-1}\right)$, it is readily seen that for each $|z|>1, Q$ admits one rightgoing wave $z^{n} \kappa^{j}$ and one leftgoing wave $z^{n} \mu^{j}$, with $\operatorname{Re} \kappa<0<\operatorname{Re} \mu,|\kappa|<1<|\mu|$, and $\mu=-1 / \kappa$. The first inequality is derived as follows in Theorem 2.4.1 of [24]. If $Q$ is $A$-stable, then $\operatorname{Re}(\kappa-1 / \kappa) \leqslant 0$ implies $|z| \leqslant 1$. Contrapositively, $|z|>1$ implies $\operatorname{Re}(\kappa-1 / \kappa)>0$. Since $|\kappa|<1$, this means that $|z|>1$ implies $\operatorname{Re} \kappa<0$.

Now we compute reflection coefficients. At $j=\Delta j+\frac{1}{2}$ one has

$$
A_{2}=-\sqrt{\kappa / \mu}=-i \kappa,
$$

and at $j=\frac{1}{2}$,

$$
A_{1}=-\sqrt{\kappa / \mu}\left(\frac{\mu-1}{\kappa-1}\right)^{q}=-i \kappa^{1-q}\left(\frac{1+\kappa}{1-\kappa}\right)^{q} \text {. }
$$

By the above inequalities one has $|(1+\kappa) /(1-\kappa)|<1$ for $|z|>1$, and therefore

$$
\left|A_{2}\right|<1, \quad\left|A_{1}\right|<|\kappa|^{1-q} \text {. }
$$

For $q=1$ both reflection coefficients have magnitude $<1$, and by the argument of Proposition 5 we are done. If $q>1$, the assumption $\Delta j+1 \geqslant q$ implies that the term $K^{\Delta j}$ in (2.11) cancels any amplification due to the factor $|\kappa|^{1-q}$ above, so stability follows from Lemma 1 . Alternatively, to stick with the one-boundary-at-atime approach of Proposition 5, one can renumber the vertices so that the left-hand boundary lies at $j=q-\frac{1}{2}$ instead of $j=\frac{1}{2}$, and then $\left|A_{1}\right|$ will be $<1$ regardless of $q$.

Remark. A similar argument can be applied to the LF model (1.8) together with a space-time extrapolation condition such as $v_{0}^{n+1}=v_{1}^{n}$.

3. Two Interfaces Separated By a Fixed Distance $\Delta x$. In this section we continue to investigate the configuration illustrated in Figure 5, except that $\Delta x$ rather than $\Delta j$ will be held constant. Consider a two-interface model $\bar{Q}=Q_{-}\left|Q_{0}\right| Q_{+}$in which the interfaces lie at positions $x_{1}=j_{1} h$ and $x_{2}=j_{2} h$, and set $\Delta x=x_{2}-x_{1}$. Either or both of the interfaces may in fact be a boundary; if both of them are, then $\bar{Q}$ is a model for a differential equation on a strip such as $[0,1]$. We ask: as the mesh is refined, i.e., as $h, k \rightarrow 0$ with $x_{1}$ and $x_{2}$ fixed, will the behavior of $\bar{Q}$ be stable or unstable?

It is now that the distinction between stability and $P$-stability becomes important. Following Beam, Warming, and Yee [1], define:

Definition. The fixed- $\Delta x$ two-interface model $\bar{Q}$ described above is $P$-stable if it is GKS-stable and, in addition, for each fixed $h>0$, it admits no solutions $v_{j}^{n}=z^{n} \phi_{j}$ with $|z|>1$ containing only leftgoing waves to the left and rightgoing waves to the right of both interfaces.

(" $P$ " stands for "practical".) Actually, $P$-stability is not a stability concept of the usual sort, since it is defined in terms of what eigensolutions $\bar{Q}$ admits rather than what growth estimate it satisfies. But obviously, this condition is vital if the time-dependent finite-difference model is to be used to approximate steady-state solutions, a procedure that is common in practice. In their tests Beam, et al. found $P$-stability of a linearized model problem to be a good indicator of success in practical nonlinear steady-state flow calculations [31]. 
We begin with the following result due to Kreiss:

Proposition 6. The fixed- $\Delta x$ two-interface model described above is GKS-stable if and only if both interfaces $Q_{-} \mid Q_{0}$ and $Q_{0} \mid Q_{+}$are individually $G K S$-stable.

Proof. See Section 11 of [10] and also Section 2 of [12]. The result refers specifically to GKS-stability, and is not necessarily valid for other definitions such as $l_{2}$-stability. The basis of the argument is the invariance of GKS-stability with respect to perturbations of size $O(k)$; the effect of each boundary on the other can be shown to be of this order as $h, k \rightarrow 0$.

The conclusion of Proposition 6 corresponds to what is often observed in practice: if each of two interfaces is GKS-stable, the computational results are usually satisfactory, while if one of them is not, they are usually wrong and sometimes explosively so. But this section can be viewed as an investigation of how Proposition 6 fails to tell the whole story. Our remaining results can be summarized as follows. Proposition 7 shows that repeated reflections between GKS-stable interfaces can cause $P$-unstable growth at a rate const ${ }^{t}$, even though GKS-stability is maintained (cf. [1] and Section 7 of [10]). Proposition 8 shows that reflection between weakly GKS-unstable interfaces can cause catastrophic growth at the rate $(\Delta j)^{\text {const } t}$ (cf. Section 17 of [14]). Proposition 9 shows that the latter problem will not occur when the unstable interfaces have finite reflection coefficients. Proposition 10, like Proposition 5, shows that all growth can be ruled out if the numerical reflection matrices satisfy $\left\|A_{1}\right\|\left\|A_{2}\right\|<1$. Finally, Proposition 11 shows that in the case of dissipative models, for the last conclusion it is enough to consider the reflection matrices $\tilde{A}_{1}, \tilde{A}_{2}$ for the differential equation itself.

Proposition 7. GKS-stability does not imply P-stability. Specifically, let each interface in the fixed- $\Delta x$ two-interface model described above be GKS-stable. If the reflection matrix at one or both interfaces has norm greater than 1 , then repeated reflections between the interfaces may sometimes lead to solution growth at the rate

$$
\left\|v^{n}\right\| \geqslant(\text { const })^{t}\left\|v^{0}\right\|
$$

Proof. In the following discussion, we first explain the growth rate const ${ }^{t}$ by two different heuristic arguments, which will be used again later in this section. The purpose of these arguments is to show that, although growth at the rate (3.1) need not occur for every model satisfying the hypotheses, it is nevertheless the typical growth rate to be expected in such problems. The proof of the proposition as stated then consists of exhibiting Example 4.

Argument by repeated reflections. The principle of Proposition 7 is the same as that of Figure 4, except that $\Delta x$ rather than $\Delta j$ is held constant. Suppose that for some $|z|=1$, a (nondissipating) wave of frequency $z$ exists which can travel leftwards with $C<0$, reflect at the $Q_{-} \mid Q_{0}$ interface into a rightgoing wave with $C>0$, and then reflect at the $Q_{0} \mid Q_{+}$interface into the original leftgoing wave mode again. If the product of the two reflection coefficients in this circuit is greater than 1 , then amplification has taken place, and it will be repeated in further reflections. The time taken to complete each circuit is roughly constant, independent of $h$ and $k$ as $h, k \rightarrow 0$. Therefore, one must expect growth at the rate const ${ }^{t}$. 
Argument by perturbed reflection coefficients. If $\bar{Q}$ permits geometric growth in $t$, we can expect the existence of an eigensolution $v_{j}^{n}=z_{0} \phi_{j}$ with $\left|z_{0}\right|>1$; the rate of growth will depend on how large $\left|z_{0}\right|$ can be. For simplicity, suppose that $Q_{0}$ admits just one rightgoing mode $z^{n} \kappa^{j}$ and one leftgoing mode $z^{n} \mu^{j}$ for each $|z| \geqslant 1$, and as in the above argument, suppose that for some $\left|z_{0}\right|=1$ one has $|\kappa|=|\mu|=1$, $C_{\mu}<0<C_{\kappa}$, and $\left|A_{1} A_{2}\right|>1$. Then the diagonal matrices $K$ and $M$ of Section 2 reduce to $\kappa$ and $\mu$, and the matrix $E_{L}$ of (2.11) is a scalar with modulus $\left|A_{1} A_{2}\right|$. Obviously, this scalar is not equal to 1 , so by Lemma $1, \bar{Q}$ does not have a solution $z_{0}^{n} \phi_{j}$. But suppose it happens that $E_{L}=1+$ const, where, here and from now on, const denotes a quantity of order of magnitude 1 that varies from one occurrence to the next and is positive except possibly for an imaginary part of size $O(\varepsilon)$, when this makes sense in context. To find a solution satisfying (2.7), consider $z=z_{0}(1+\varepsilon)$, $0<\varepsilon \ll 1$. This perturbation changes $\kappa, \mu, A_{1}$ and $A_{2}$ by $O(\varepsilon)$. In particular, $\kappa$ and $\mu$ become

$$
\kappa \rightarrow \kappa(1-\text { const } \varepsilon), \quad \mu \rightarrow \mu(1+\text { const } \varepsilon) .
$$

(In the limit $\varepsilon=0$, the constants here are $1 / \lambda\left|C_{\kappa}\right|$ and $1 / \lambda\left|C_{\mu}\right|$. ) By (2.11), $E_{L}$ therefore becomes

$$
E_{L}=(1+\text { const })(1-\text { const } \varepsilon)^{\Delta j} \text {. }
$$

For $E_{L}$ to have value 1 , the two factors have to balance, which means $\varepsilon=O(1 / \Delta j)$. Therefore, one can expect that any eigensolution $z^{n} \phi_{j}$ to $\bar{Q}$ will grow at the rate

$$
\frac{\left\|v^{n}\right\|}{\left\|v^{0}\right\|} \approx|z|^{n}=\left(1+\frac{\text { const }}{\Delta j}\right)^{n}=\left(1+\frac{\text { const }}{\Delta j}\right)^{t{ }_{j}}=\text { const }^{t},
$$

as asserted in (3.1).

Example 4. Let $u_{t}=u_{x}$ on $[0,1]$ be approximated by the LF formula (1.8) together with the (admittedly contrived) boundary conditions

$$
v_{0}^{n+1}=\frac{1}{2}\left(v_{2}^{n-1}+v_{3}^{n-2}\right), \quad v_{\Delta j+1}^{n+1}=0 .
$$

The reflection coefficient functions are easily seen to be

$$
A_{1}=-\sqrt{\kappa / \mu} \frac{2 z^{3}-z \mu^{2}-\mu^{3}}{2 z^{3}-z \kappa^{2}-\kappa^{3}}, \quad A_{2}=-\sqrt{\kappa / \mu},
$$

and since the denominators are never zero, both interfaces are GKS-stable. However, $\left|A_{1}\right|$ can be larger than 1 . For simplicity, consider the semidiscrete limit $\lambda=0$, $z=1$. By (1.17), for any $\theta=\xi h \in[0, \pi / 2)$, LF then has a solution

$$
z=1, \quad \mu=e^{i \theta}, \quad \kappa=-e^{-i \theta}, \quad C_{\mu}=-\cos \theta, \quad C_{\kappa}=\cos \theta .
$$

For any $\theta$ with $\left|A_{1}(\theta)\right|>1$, one can expect $\bar{Q}$ to admit an eigensolution that grows approximately at the rate $\left|A_{1}(\theta)\right|^{t \cos \theta / 2}$, since each circuit of a trapped wave packet will take time $2 / \cos \theta$. The maximum of these rates for the given formulas turns out to be at $\theta \approx .75$, where one gets $\left|A_{1}\right| \approx 2.38, C \approx .725$, and growth (1.37) ${ }^{t}$. Numerical experiments confirm that solutions grow roughly at this rate, independently of $h$ and $k$. 
To establish Proposition 7 rigorously, one must prove that $\bar{Q}$ admits the kind of growing eigensolution we have described. This can be done by using perturbation arguments based on the above heuristic reasoning to show that (2.11) in Lemma 1 has a solution with $|z| \approx(1.37)^{k}$. Since the conclusion is so obvious, we will not give details.

The possibility of $P$-unstable growth as in Example 4 was recognized from the start by Gustafsson, Kreiss, and Sundström, and in fact Section 7 of [10] is devoted to determining when it will occur in a certain $2 \times 2$ problem. In our particular example, the model remains $P$-unstable no matter how small $h$ and $k$ become. Beam, et al. give the impression in various papers that this cannot happen, but that is true only when one is dealing with dissipative formulas; see Proposition 10 below. The reason that dissipation did not ensure $P$-stability for the values of $h$ and $k$ they were dealing with was that, because of their interest in steady-state results, they were using very large values of $\lambda$, and their formulas happened to be nondissipative in the limit $\lambda \rightarrow \infty$. Thus, their computations made use of difference formulas that were dissipative but only weakly so.

Now, let reflection coefficients be present that are not merely greater than 1 , but infinite. The potential growth rate becomes much more severe.

Proposition 8. Let one or both interfaces in the fixed- $\Delta x$ two-interface problem be algebraically GKS-unstable, with an infinite reflection coefficient. Then repeated reflections between the interfaces may sometimes lead to solution growth at the exponential rate

$$
\left\|v^{n}\right\| \geqslant(\Delta j)^{\text {const } t}\left\|v^{0}\right\|
$$

Remark. For a single GKS-unstable interface with an infinite-reflection coefficient, it is shown in [27] that the unstable growth is in general no worse than $\left\|v^{n}\right\| \approx$ const $n\left\|v^{0}\right\|$. This is what is meant above by "algebraically" GKS-unstable.

Proof. Again we will motivate (3.4) by two arguments. Then we prove the proposition by exhibiting Example 5.

Argument by repeated reflections. Suppose $A_{1}$ is infinite at $z=z_{0}$, and behaves near there like

$$
\left\|A_{1}\right\|=\frac{\text { const }}{\left|z-z_{0}\right|}
$$

Since there are only $\Delta j$ points between the interfaces for each fixed $h$, Fourier analysis implies that no wave on the $Q_{0}$ grid can have a spectrum narrower than $O(1 / \Delta j)$. Therefore, it is plausible that in applying (3.5) to the finite grid, the largest amplification possible will be that obtained with an effective value $z_{\text {eff }}$ with $\left|z_{\text {eff }}-z_{0}\right|=$ const $/ \Delta j$, i.e., $\left\|A_{1}\right\| \approx$ const $\Delta j$. Since as before each circuit takes roughly a fixed amount of time, independent of $h$ and $k$ as $h, k \rightarrow 0$, this leads immediately to (3.4).

Argument by perturbed reflection coefficients. As before, suppose that $Q_{0}$ admits one leftgoing mode $z^{n} \mu^{j}$ and one rightgoing mode $z^{n} \kappa^{j}$ for each $|z| \geqslant 1$, and that for some $\left|z_{0}\right|=1$ one has $|\kappa|=|\mu|=1, C_{\mu}<0<C_{\kappa},\left|A_{1}\left(z_{0}\right)\right|=\infty$, and $\left|A_{2}\left(z_{0}\right)\right|>0$. 
Suppose, furthermore, that $A_{1}$ behaves like (3.5) for $z \approx z_{0}$. Then, under the perturbation $z=z_{0}(1+\varepsilon)$, one has

$$
\kappa \rightarrow \kappa(1-\operatorname{const} \varepsilon), \quad \mu \rightarrow \mu(1+\text { const } \varepsilon), \quad E_{L} \rightarrow \frac{(1-\text { const } \varepsilon)^{\Delta j}}{\varepsilon}
$$

For $E_{L}=1$ we must have $(1-\text { const } \varepsilon)^{\Delta j}=\varepsilon$, which implies

$$
\varepsilon \approx \frac{\text { const }}{\Delta j} \log (\Delta j) \text {. }
$$

Hence, growth should be expected at the rate

$$
\frac{\left\|v^{n}\right\|}{\left\|v^{0}\right\|}=|z|^{n}=\left(1+\frac{\text { const } \log \Delta j}{\Delta j}\right)^{t \Delta j}=e^{t \text { const } \log \Delta j}=(\Delta j)^{\text {const } t} .
$$

Example 5 ([14, Section 17]). Let $u_{t}=u_{x}$ on $[0,1]$ be approximated by LF (1.8) with boundary conditions $v_{0}^{n+1}=v_{1}^{n+1}(1.16)$ and $v_{\Delta j+1}^{n+1}=0$. We have seen in (1.18) that this model has an infinite reflection coefficient at $z=-1$; in fact one has as in Example 3,

$$
A_{1}(z)=-\sqrt{\kappa / \mu} \frac{1-\mu}{1-\kappa}, \quad A_{2}=-\sqrt{\kappa / \mu} .
$$

With these formulas (2.11) becomes

$$
E_{L}(z)=(\kappa / \mu)^{\Delta j+1} \frac{1-\mu}{1-\kappa},
$$

and since $\mu=-1 / \kappa$ for $L F$, this can be rewritten

$$
E_{L}(z)=\left(-\kappa^{2}\right)^{\Delta j+1} \frac{1+1 / \kappa}{1-\kappa} .
$$

Assume $\Delta j+1$ is even, and write $\kappa=1-\delta$. The condition $E_{L}(z)=1$ becomes

$$
(1-\delta)^{2 \Delta_{j}+2}=\frac{\delta-\delta^{2}}{2-\delta}
$$

It is obvious that this equation has a positive real solution near $\delta=0$, which is asymptotic to $\delta=\log \Delta j / 2 \Delta j$ as $\Delta j \rightarrow \infty$. The corresponding value of $z$ is asymptotic to

$$
z=1+\frac{\lambda}{2} \frac{\log \Delta j}{\Delta j}
$$

Therefore, $\bar{Q}$ has an eigensolution which grows at the rate

$$
\left(1+\frac{\lambda}{2} \frac{\log \Delta j}{\Delta j}\right)^{n}=\left(1+\frac{\lambda}{2} \frac{\log \Delta j}{\Delta j}\right)^{t \Delta j / \lambda} \approx e^{t \log \Delta j / 2}=(\Delta j)^{t / 2} .
$$

This matches the result stated as (17.10) in [14], and numerical experiments confirm that physical solutions are rapidly obliterated by growth at the predicted rate.

The possibility of catastrophic two-boundary interactions as in Proposition 8 has long been recognized by Kreiss and his colleagues, and it has been given sometimes as a justification of the apparent strictness of the GKS stability definition. We now 
show that this justification is only partial, for not all GKS-unstable boundaries have infinite reflection coefficients, yet an infinite reflection coefficient is required for the catastrophic two-boundary interaction to occur:

Proposition 9. Let one or both interfaces in the fixed- $\Delta x$ two-interface problem be algebraically GKS-unstable, but with finite reflection coefficients only. Then $\bar{Q}$ admits no eigensolutions $v_{j}^{n}=z^{n} \phi_{j}$ that grow faster than (const) ${ }^{t}$.

Proof. Consider an eigensolution $z^{n} \phi_{j}$ of $\bar{Q}$, and let $M, K, A_{2}, A_{1}$ be the matrices of Lemma 1 for the given value $z$. By Lemma 1 , the matrix

$$
E_{L}=M^{-{ }^{\Delta j}} A_{2} K^{\Delta j} A_{1}
$$

has an eigenvalue 1 , which implies $\left\|E_{L}\right\|_{\infty} \geqslant 1$. On the other hand, the finite reflection coefficients assumption implies

$$
\left\|A_{1}\right\|_{\infty}\left\|A_{2}\right\|_{\infty} \leqslant T
$$

for some $T<\infty$. These bounds together yield

$$
\left\|M^{-1}\right\|_{\infty}^{\Delta j}\|K\|_{\infty}^{\Delta j} \geqslant 1 / T
$$

or in particular, since $|\kappa| \leqslant 1 \leqslant|\mu|$ for each of the entries in $M$ and $K$,

$$
|\kappa|,\left|\mu^{-1}\right| \geqslant(1 / T)^{1 / \Delta j}
$$

for some $\kappa$ and $\mu$.

Now the critical observation is that for any Cauchy stable formula $Q,|z|-1$ is bounded by a multiple of $1-|\kappa|$ when the latter is small. For a proof, see Lemma 9.1 of [10]; the constant factor is essentially $\lambda$ times the maximum group velocity admitted by $Q$. Therefore, the last inequality implies $|z| \leqslant T^{\text {const } / \Delta j}$ for large enough $\Delta j$. But this leads to

$$
|z|^{n} \leqslant(T)^{n \text { const/ } / \Delta_{j}}=\text { const }^{t},
$$

which proves the proposition.

Our next result is the same as Proposition 5, but restated for the fixed- $\Delta x$ problem.

PROPOSITION 10. In the fixed- $\Delta x$ two-interface problem, in which each interface individually is GKS-stable, a sufficient condition for P-stability is

$$
\left\|A_{1}(z)\right\|\left\|A_{2}(z)\right\|<1 \text { for all }|z| \geqslant 1
$$

in any norm subordinate to a vector norm.

Proof. Same as for Proposition 5.

Finally, we return to the question of dissipation. In the fixed- $\Delta j$ situation, the use of dissipative formulas gave no guarantee of stability, because the attenuation introduced by dissipation might always be overcome by amplification due to reflection at the boundaries. But in the fixed- $\Delta x$ problem, the attenuation of any nonphysical wave mode will increase as the mesh is refined. For fine enough meshes, this must overcome any finite amplification factors. 
To obtain a theorem along these lines, though not the sharpest possible, assume for simplicity as in [10] that the matrix $A$ in the differential equation (1.1) is already diagonal, with $\tilde{L}$ positive and $\tilde{R}=N-\tilde{L}$ negative eigenvalues, and let $\tilde{A}_{1}(\tilde{R} \times \tilde{L})$ and $\tilde{A}_{2}(\tilde{L} \times \tilde{R})$ be reflection matrices imposed as homogeneous boundary conditions to define an initial-boundary value problem for (1.1) on $\left[x_{1}, x_{2}\right]$. Then we have

Proposition 11. Let $\bar{Q}$ be a GKS-stable, totally dissipative, consistent model of the fixed- $\Delta x$ two-interface problem described above, and suppose

$$
\left\|\tilde{A}_{1}\right\|\left\|\tilde{A}_{2}\right\|<1
$$

in some norm subordinate to a vector norm. Then, for all sufficiently small $h$ and $k, \bar{Q}$ is P-stable.

Remark. By "totally dissipative," we mean that the interior model $Q$ dissipates oscillations with respect to $t$ as well as $x$. For two-level formulas this is the same as the usual definition of dissipativity. For multi-level formulas, there is the additional requirement that the scheme admit no solutions $v_{j}^{n}=z^{n} \phi, \phi=$ const, with $|z|=1$ but $z \neq 1[24]$.

Remark. This result is related to the theorem stated by Gustafsson in [11]. See also [7].

Proof. We must show that $\bar{Q}$ admits no eigensolution $z^{n} \phi_{j}$ with $|z|>1$, for large enough $\Delta j$. Suppose to the contrary that for a sequence of values $\Delta j \rightarrow \infty, \bar{Q}$ has a solution $z^{n} \phi_{j}$ with $|z|>1$. Since $\bar{Q}$ is GKS-stable, it has finite reflection coefficients, so (3.6)-(3.8) of the last proof are again valid. Equation (3.8) implies $|\kappa| \uparrow 1$ and $|\mu| \downarrow 1$ for some $\kappa$ and $\mu$ as $\Delta j \rightarrow \infty$. By dissipativity, this implies $\kappa \rightarrow 1$ and $\mu \rightarrow 1$. By total dissipativity, this in turn implies $z \rightarrow 1$ also.

Consider the behavior of the $L \times L$ matrix $E_{L}$ of (2.11) as $\Delta j \rightarrow \infty$ and $z \rightarrow 1$. By consistency, $\tilde{L}$ values $\mu$ and $\tilde{R}$ values $\kappa$ approach 1 , and the corresponding basis vectors in terms of which $E_{L}$ is defined approach the basis vectors for the differential equation, namely unit vectors of the form $(0, \ldots, 0,1,0, \ldots, 0)^{T}$. Consistency further implies that the numerical reflection matrices $A_{1}$ and $A_{2}$, when restricted to these $\tilde{L}$ and $\tilde{R}$ rows and columns, approach $\tilde{A}_{1}$ and $\tilde{A}_{2}$. On the other hand, the remaining $L-\tilde{L}$ values $\mu$ and $R-\tilde{R}$ values $\kappa$ are bounded away from 1 in modulus as $\Delta j \rightarrow \infty$, and therefore the elements of $M^{-\Delta_{j}}$ and $K^{\Delta_{j}}$ in these remaining positions converge to zero as $\Delta j \rightarrow \infty$. These observations imply

$$
\varlimsup_{\Delta j \rightarrow \infty}\left\|K^{\Delta_{j}} A_{1}\right\| \leqslant\left\|\tilde{A}_{1}\right\|, \quad \varlimsup_{\Delta j \rightarrow \infty}\left\|M^{-\Delta^{j}} A_{2}\right\| \leqslant\left\|\tilde{A}_{2}\right\| .
$$

(The norms on the matrices without tildes are arbitrary, so long as they reduce to the norms on the matrices with tildes when restricted to the appropriate components.) Together with the hypothesis, these bounds show that $E_{L}$ cannot have 1 as an eigenvalue. Therefore, by Lemma 1 , the assumed sequence of eigensolutions cannot exist after all.

Acknowledgements. I am grateful for valuable discussions with Robert Warming and Michael Giles. Marsha Berger made many constructive suggestions and caught an error in the formulation of Proposition 5. 


\begin{tabular}{ll} 
& \multicolumn{1}{c}{$\begin{array}{c}\text { APPENDIX } \\
\text { Notation }\end{array}$} \\
$j, n$ & space, time index \\
$h, k$ & space, time step size \\
$\lambda=k / h$ & mesh ratio \\
$u(x, t), v_{j}^{n}$ & continuous, discrete solution vector \\
$N$ & dimension of $u, v$ \\
$\xi, \omega$ & wave number, frequency \\
$C(\xi, \omega)$ & group velocity \\
$z$ & temporal amplification factor \\
$\kappa, \mu$ & rightgoing, leftgoing spatial amplification factor \\
$l, r$ & no. of points to the left, right of center in stencil \\
$R=N l, L=N r$ & no. of rightgoing, leftgoing numerical wave modes \\
$Q, \bar{Q}$ & finite difference model for i.v.p., i.b.v.p. \\
$A(z)$ & reflection matrix function at boundary \\
const & positive constant, different each time
\end{tabular}

Department of Mathematics

Massachusetts Institute of Technology

Cambridge, Massachusetts 02139

1. R. M. BeAm, R. F. Warming \& H. C. YeE, Stability Analysis for Numerical Boundary Conditions and Implicit Difference Approximations of Hyperbolic Equations, Proc. NASA Sympos. on Numerical Boundary Procedures, 1981, pp. 1.99-207.

2. M. Berger, Adaptive Mesh Refinement for Hyperbolic Partial Differential Equations, Ph. D. Thesis, Department of Computer Science, Stanford University, 1982.

3. M. Berger, Stability of Interfaces with Mesh Refinement, ICASE Report 83-42.

4. D. BROWN, "A note on the numerical solution of the wave equation with piecewise smooth coefficients," Math. Comp., v. 42, 1984, pp. 369-391.

5. G. Browning, H.-O. Kreiss \& J. Oliger, "Mesh refinement," Math. Comp., v. 27, 1973, pp. 29-39.

6. M. Ciment, "Stable matching of difference schemes," SIAM J. Numer. Anal., v. 9, 1972, pp. 695-701.

7. M. Giles, Eigenmode Analysis of Unsteady One-Dimensional Euler Equations, NASA Contract Report 172217, ICASE 1983, submitted to AIAA J.

8. M. Giles \& W. Thompkins, Jr., Asymptotic Analysis of Numerical Save Propagation in Finite Difference Equations, Gas Turbine and Plasma Phys. Lab. Rep. 171, Massachusetts Institute of Technology, 1983.

9. M. GOLDBERG \& E. TADMOR, "Scheme-independent stability criteria for difference approximations of hyperbolic initial-boundary value problems. II," Math. Comp., v. 36, 1981, pp. 603-626.

10. B. GustafsSON, H.-O. KREISS \& A. SundSTROM, "Stability theory of difference approximations for initial boundary value problems. II," Math. Comp., v. 26, 1972, pp. 649-686.

11. B. Gustafsson, The Choice of Numerical Boundary Conditions for Hyperbolic Systems, Proc. NASA Ames Sympos. on Numerical Boundary Procedures, 1981, pp. 209-225.

12. H.-O. KREISS, "Difference approximations for the initial-boundary value problem for hyperbolic differential equations," in Numerical Solution of Nonlinear Differential Equations (D. Greenspan, ed.), Proc. Advanced Symposium Mathematics Research Center, University of Wisconsin, pp. 141-166, Wiley, New York, 1966

13. H.-O. KreIss, "Initial boundary value problems for hyperbolic systems," Comm. Pure Appl. Math., v. 23,1970 , pp. $277-298$.

14. H.-O. Kreiss \& J. Oliger, Methods for the Approximate Solution of Time Dependent Prohlems. Global Atmospheric Research Programme Publ. Series No. 10, Geneva, 1973. 
15. J. OLIGER, "Fourth-order difference methods for the initial boundary-value problem for hyperbolic equations," Math. Comp., v. 28, 1974, pp. 15-25.

16. J. Oliger, "Hybrid difference methods for the initial boundary-value problem for hyperbolic equations," Math. Comp., v. 30, 1976, pp. 724-738.

17. J. Oliger, "Constructing stable difference methods for hyperbolic equations," Numerical Methods for Partial Difference Equations (S. Parter, ed.), Academic Press, New York, 1979.

18. S. OSHER, "Systems of difference equations with general homogeneous boundary conditions," Trans. Amer. Math. Soc., v. 137, 1969, pp. 177-201.

19. S. OSHER, "Hyperbolic equations in regions with characteristic boundaries or with corners," Numerical Solution of Partial Differential Equations III (B. Hubbard, ed.), Academic Press, New York, 1976.

20. R. Richtmyer \& K. Morton, Difference Methods for Initial-Value Problems, Wiley-Interscience, New York, 1967.

21. L. Sarason \& J. A. SMOller, "Geometrical optics and the corner problem," Arch. Rational Mech. Anal., v. 56, 1975, pp. 34-69.

22. J. C. South \& M. M. HAFEZ, Stability Analysis of Intermediate Boundary Conditions in Approximate Factorization Schemes, Proc. 6th AIAA Computational Fluid Dynamics Conference, Danvers, MA, 1983.

23. J. A. TRAPP \& J. D. RAMSHAW, "A simple heuristic method for analyzing the effects of boundary conditions on numerical stability," J. Comput. Phys., v. 20, 1976, pp. 238-242.

24. L. N. Trefethen, Wave Propagation and Stability for Finite Difference Schemes, Ph. D. Thesis, Department of Computer Science, Stanford University, 1982.

25. L. N. Trefethen, "Group velocity in finite difference schemes," SIAM Rev., v. 24, 1982, pp. $113-136$.

26. L. N. TREFETheN, "Group velocity interpretation of the stability theory of Gustafsson, Kreiss, and Sundström,” J. Comput. Phys., v. 49, 1983, pp. 199-217.

27. L. N. TREFETHEN, "Instability of difference models for hyperbolic initial boundary value problems," Comm. Pure Appl. Math., v. 37, 1984, pp. 329-367.

28. L. N. Trefethen, Stability of Hyperbolic Finite-Difference Models With One or Two Boundaries, Proc. AMS-SIAM Summer Seminar, La Jolla, CA, 1984.

29. E. TURKel, "Progress in computational physics," Comput. \& Fluids, v. 11, 1983, pp. 121-144.

30. R. Vichnevetsky \& J. B. Bowles, Fourier Analysis of Numerical Approximations of Hyperbolic Equations, SIAM, Philadelphia, PA, 1982.

31. H. C. YeE, R. M. BeAm \& R. F. WARMing, Stable Boundary Approximations for a Class of Implicit Schemes for the One-Dimensional Inviscid Equations of Gas Dynamics, AIAA 5th Computational Fluid Dynamics Conference, 1981. 